\title{
Cost-effectiveness analysis of Dapagliflozin versus Canagliflozin in treatment of type 2 diabetes mellitus
}

\author{
Sadagoban Gopal Krishnamoorthy (D), Jemi Rachel Shaji, Bhagya Premnath, Akshitha Rani Siby, Swathi Swaroopa Borra (D), \\ Ayilya Manomohan, Arun Kanniyappan Parthasarathy* \\ Department of Pharmacy Practice, JSS College of Pharmacy, JSS Academy of Higher Education \& Research, Ooty, Nilgiris, Tamil Nadu, India.
}

\begin{tabular}{l}
\hline ARTICLE INFO \\
\hline Received on: $17 / 08 / 2021$ \\
Accepted on: $25 / 10 / 2021$ \\
Available Online: 05/03/2022 \\
\hline Key words: \\
Health outcome research, \\
CEA of Canagliflozin, CEA of \\
Dapagliflozin, cost effective \\
analysis, pharmacoeconomic \\
analysis.
\end{tabular}

\begin{abstract}
The objective of this study was to provide cost-effectiveness evidence regarding the use of Dapagliflozin $10 \mathrm{mg}$ and Canagliflozin $300 \mathrm{mg}$ for treating adult patients with type $2 \mathrm{DM}$ by measuring the clinical outcomes of the selected treatment regimens and by conducting a decision analysis. The main considered outcomes were cost, probabilities of diabetic complications, life year (LY), quality-adjusted life year (QALY), and incremental cost effectiveness ratio. Oneway deterministic sensitivity analysis was also carried out. Cost effective analysis was carried out and Dapagliflozin was found to be more effective and less expensive which apparently falls on Q4 North West Position. In this study, better results were found with the control group (A) Dapagliflozin than the comparison group (B) Canagliflozin. We assume that the economical and clinical outcomes of Pharmacoeconomics were affected by various other factors like medication adherence, affordability, appropriateness of regimens, and other social and cultural factors. Further studies should be conducted on these matters to understand and improve the QALY and also to reduce the burden of disease of type 2 diabetic patients.
\end{abstract}

\section{INTRODUCTION}

India is known as the diabetes hub of the world. In India, the reports claim that almost 62.4 million people are affected with diabetes and 77.2 million people are affected with pre-diabetes (Anjana et al., 2011). The average cost of the treatment per diabetic patient with or without co-existing medical conditions was found to be USD 314.15 ( $₹ 23,170.43$ ) and USD 29.91 (₹2,206.04), respectively. The average cost of treatment for the patients with macrovascular complications is USD 125.01 (₹9,220.23) and USD 90.43 (₹6,669.75) for microvascular complications (Akari et al., 2013). The demand and the cost of health care settings are increasing at an unprecedented rate. The hiking price of medicines all over the world has been affecting the patients. Approximately $85 \%$ of total health expenditure is supported by

\footnotetext{
*Corresponding Author

Arun Kanniyappan Parthasarathy, Department of Pharmacy Practice, JSS College of Pharmacy, JSS Academy of Higher Education \& Research, Ooty, India.E-mail: kparun@jssuni.edu.in
}

household out of pocket payments in a developing country like India. Due to an increased emphasis on efficient drug therapies for a disease that increases health costs, over the past 20 years the field of Pharmacoeconomics has become integral for decisionmaking policies. Metformin is considered as the initial choice for treating hyperglycemia to reduce the risk of complications related to type 2 diabetes mellitus. However, over time it may or may not adequately control hyperglycemia due to disease progression which necessitates an add-on therapy or change of drug (Hou et al., 2019). By enhancing the excretion of glucose in the urine, SGLT2 inhibitors have been accepted as the latest class of therapies for the treatment of type 2 DM (Li et al., 2017).

SGLT2 inhibitors offer cardiovascular and renal benefits, it also causes body weight loss via glucose excretion in the kidneys (Pereira and Eriksson, 2019; Rabizadeh et al., 2019). There have been only few reports on cost-effectiveness evaluations of SGLT2 inhibitors. The objective of this study is to provide costeffectiveness evidence regarding the use of Dapagliflozin $10 \mathrm{mg}$ and Canagliflozin $300 \mathrm{mg}$ for treating adult patients with type 2 
DM by measuring the clinical outcomes of the selected treatment regimens and by conducting a decision analysis.

The ECHO model which includes Economic, Clinical, and Humanistic Outcomes helps in Pharmacoeconomic analysis. Economic outcomes include direct, indirect, and intangible costs compared with the consequences of medical treatment alternatives. Clinical outcomes include medical events that occur as a result of disease or treatment. Humanistic outcomes include consequences of disease or treatment on patient functional status, or quality of life, measured along several dimensions (The Blonde Pharmacist, 2019).

\section{METHODS}

This study provides an economic analysis of Dapagliflozin and Canagliflozin for the treatment of type 2 DM. To keep with other economic reports associated with type 2 DM therapies we have used lifetime health and economic outcomes as the main end-points, which includes cost, probabilities of diabetic complications, life year (LY), quality-adjusted life year (QALY), and incremental cost effectiveness ratio (ICER).

Study design: Cost-effectiveness analysis.

Type of study: Qualitative synthesis studies.

Study site: The data for the study purpose was collected from selected community pharmacies in Kerala. (Thiruvalla, Kottayam, Kochi, and Trivandrum).

Study population: Type 2 DM patients.

Study duration: The study was carried out for 6 months.

Sample size: Purposive sampling.

\section{Inclusion criteria}

Subjects with a history of Type 2 DM minimum for 3 years. Subjects should have a minimum of 3 months of exposure to the selected treatment regimens without any interruptions. Body mass index should be between the ranges of 18.5 to $29 \mathrm{~kg} / \mathrm{m}^{2}$. Subjects with proper evidence of their past and present medical histories.

\section{Exclusion criteria}

The patients who were on insulin for controlling their hyperglycemic status. Treatment with any investigational products (e.g., Ayurveda) in the last 3 months before study entry. Patients with Diabetic Ketoacidosis are to be excluded. Patients with kidney disease are to be excluded.

\section{Ethical clearance}

The study protocol was approved by the institutional review board of the academic institution where the study was held.

\section{RESULTS}

A total of 94 patient's data were collected from the selected community pharmacies in Kerala during the specified study period, out of which 55 of them met the inclusion standards and were included in the study. The study population was divided into 31 male patients and 24 female patients. The data collected were further categorized based on the age. A total of 37 patient's data was collected from the age group of 27-55 years which accounts for the maximum number of patients who participated in the study and 18 patients belonged to the age group $>55$ years; whereas no data was acquired from the age group of 18-26 years. The mean average and standard deviation of the age of the study population was found to be $58.79 \pm 7.67$ in the Dapagliflozin treated group, $54.24 \pm 12.14$ in the Canagliflozin treated group.

The outcomes of the ECHO model were:

\section{Clinical outcome}

$\mathrm{HbA1C}$ was found to be in $7.36 \pm 0.654996$ in the Dapagliflozin treated group and $7.18 \pm 0.55$ in the Canagliflozin treated group (shown in Table 1). Both the groups achieved the
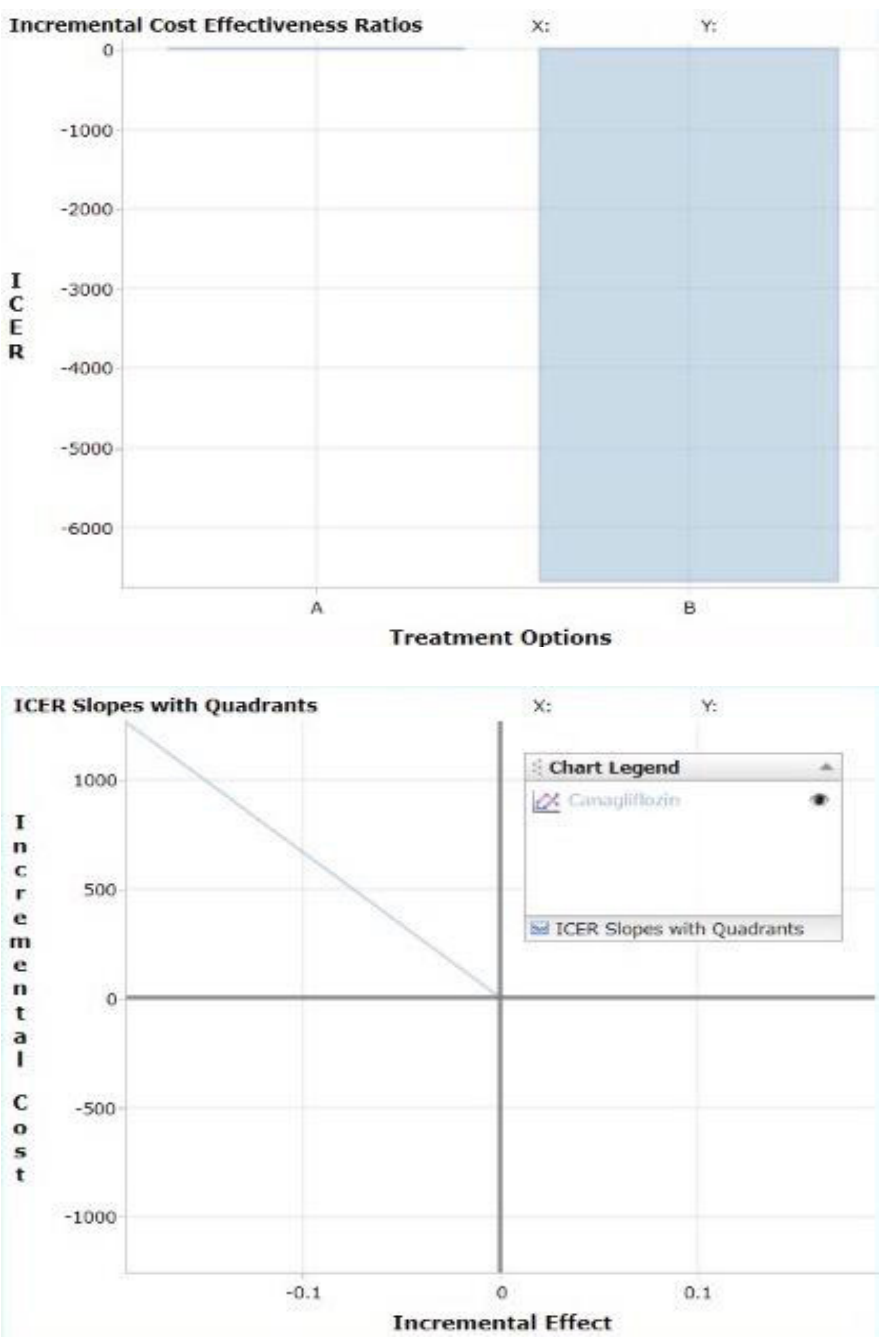

Figure 1. Bar graph and cost-effectiveness plane of type 2 DM drugs (Dapagliflozin as control group and Canagliflozin as comparison group). Negative ICER values can indicate either favorable (Accept) treatment options or unfavorable (Reject) options since the ICER value reflects a slope that can be in two different quadrants. This "Accept" or "Reject" recommendation is automatically displayed in the - calculated results-table above. See graphical explanation. It showed the control group (A) as zero and the comparison group (B) on the negative axis. 


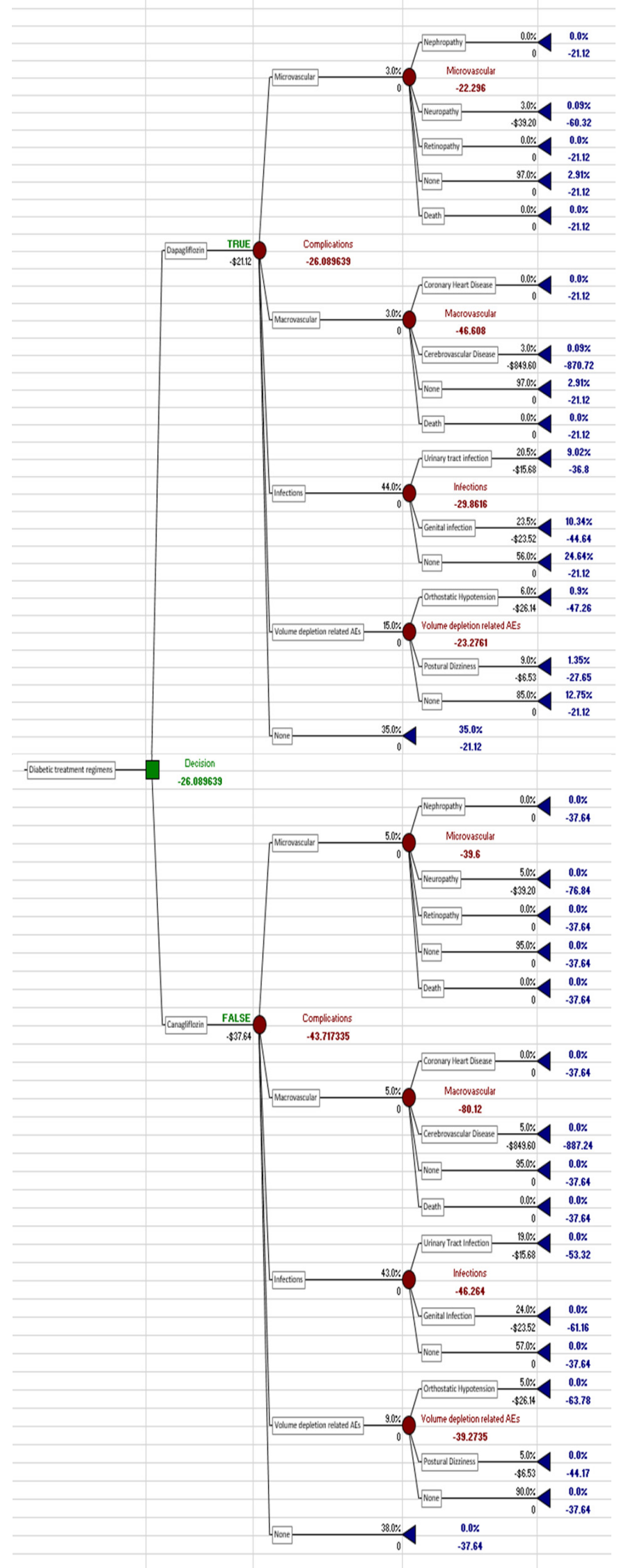

Figure 2. Decision tree analysis of Dapagliflozin and Canagliflozin. target $\mathrm{HbA1c}$ (A1C targets of $<7 \%$ or $53 \mathrm{mmol} / \mathrm{mol}$ ) but better results were shown in the Canagliflozin treated group. Duration of illness (in years) was also calculated for both treatment regimens and was found to be $9.97 \pm 4.69$ for Dapagliflozin, $7.95 \pm 2.84$ for Canagliflozin.

\section{Economic outcome}

As per WHO CHOICE recommendations, the direct costs of medications were identified by finding the mean price value per tablet. The three brands of Dapagliflozin and one brand of Canagliflozin available in India were selected for finding the mean price value (MPV). The MPV found in rupees was converted into dollars. It was found to be $\$ 0.70$ (₹53.87) in the Dapagliflozin treated group, $\$ 96.00$ (₹1.25) in the Canagliflozin treated group.

The average cost per patient per day and the average cost per patient per year were calculated both in rupees and in dollars. Both were found to be highest in Canagliflozin treated group. Based on Table 2 information the average cost per patient per year was found to be $\$ 260.224$ (₹19,918.3) in the Dapagliflozin treated group, $\$ 456.25$ (₹35,040) in the Canagliflozin treated group.

\section{Humanistic outcome}

People with diabetes can experience various discomforting physical and mental symptoms. Each of the patients were provided with a self-administering questionnaire comprising 34 questions. By using the DSC-R checklist each patient's overall health and mental wellbeing were captured. The DSC-R Mean Dimensional Score (MDS) was calculated; a total of eight domains were accounted into the analysis. MDS was found to be 1.92 for Dapagliflozin treated group and 2.29 for Canagliflozin treated group. Canagliflozin treated group showed a higher MDS score indicating more symptoms or complications in these patients.

\section{Quality-adjusted life year}

In this expected life years was assumed to be 1 . MDS was converted into a utility value to a rating scale of 0 to 1 . QALY for both treatment regimens was calculated. Dapagliflozin

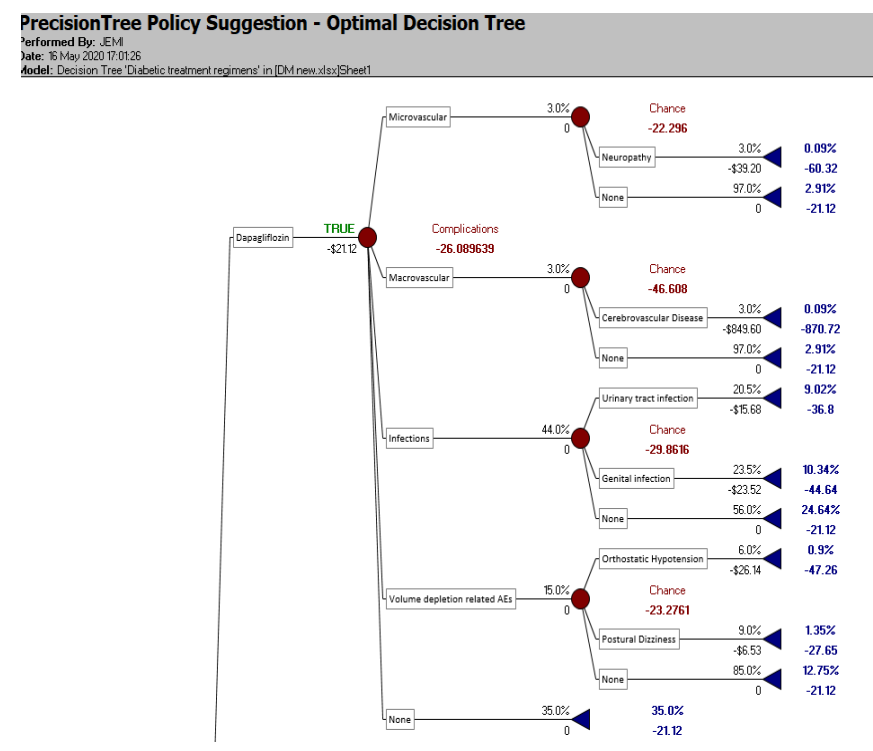

Figure 3. Optimal decision tree. 
Table 1. Clinical outcomes of Dapagliflozin and Canagliflozin

\begin{tabular}{ccccc}
\hline DRUGS & HbA1c & Duration of illness (years) & $\begin{array}{c}\text { No. of patients within normal } \\
\text { range }\end{array}$ & $\begin{array}{c}\text { No. of patients above normal } \\
\text { range }\end{array}$ \\
\hline Dapagliflozin & $7 \pm 0.65$ & $10 \pm 5$ & 14 & 20 \\
Canagliflozin & $7 \pm 0.54$ & $8 \pm 3$ & 13 & 8 \\
\hline
\end{tabular}

HbA1c- glycated haemoglobin.

Table 2. Economic outcomes of Dapagliflozin and Canagliflozin.

\begin{tabular}{cccccc}
\hline Drugs & \multicolumn{2}{c}{ Direct cost } & \multicolumn{2}{c}{ Indirect cost } & Total cost \\
\hline Per patient & $\begin{array}{c}\text { Consultation/follow up and } \\
\text { hospital admission }\end{array}$ & Drug price & Travel & Food and expenditures & \\
\hline Dapagliflozin $(n=34)$ & $₹ 3,068.52 / \$ 40.11$ & $₹ 1,616.34 / \$ 21.12$ & $₹ 222.94 / \$ 2.91$ & $₹ 117.64 / \$ 1.53$ & $₹ 5,025.44 / \$ 65.67$ \\
Canagliflozin $(n=21)$ & $₹ 4,288.57 / \$ 56.05$ & $₹ 2,880.0 / \$ 37.64$ & $₹ 230.95 / \$ 3.01$ & $₹ 156.19 / \$ 2.04$ & $₹ 7,555.71 / \$ 98.74$ \\
\hline
\end{tabular}

treated group had a QALY of 0.616, Canagliflozin treated group had a QALY of 0.543 . A QALY of 1 shows full health. Both the treatment groups showed only partial health but better results were provided by Dapagliflozin.

The SGLT-2 drugs cause significant weight reduction. The average weight reduction over 3 months was found to be 2.31 $\mathrm{kg}$ in Canagliflozin treated patients and $2.59 \mathrm{~kg}$ in Dapagliflozin treated patients.

\section{CEA (cost effective analysis) calculation for various type 2 DM treatment regimens}

CEA was calculated by comparing both the treatment groups Dapagliflozin and Canagliflozin. The control group A had an incremental cost of zero which was accepted, comparison group B had an incremental cost of ₹1,264 (shown in Table 3). The control group was accepted which showed an intersection and the comparison group showed the line to the Q4 -North West (shown in Figure 1). This shows a rejection of the comparison group due to higher cost and lower effectiveness.

\section{Decision analysis}

A decision tree model was created (shown in Figure 2) using the Palisade software. The data collected for the study purpose from various community pharmacies were fed into the software, and the data set comprised; the cost of each drug, the cost of treatment of associated complications, and the probability of developing these complications. The tree comprises two main branches each of which represents the drugs (Canagliflozin and
Dapagliflozin). The main branch was further divided into multiple child branches which represent the complications - Microvascular (Nephropathy, Neuropathy, Retinopathy) and the probability of not developing neither of these (None) and Death due to untreated complication. Macrovascular (Coronary Heart Disease, Cerebrovascular Disease, None and Death). The infections were further branched into urinary tract infections, Genital Infections and None. Volume depletion related AEs were further classified into Orthostatic Hypotension, Postural Dizziness, and None. After considering multiple factors like the cost of the drug, cost of treating these complications and probability of acquiring these complications the software in itself generates an optimal outcome that aids the decision-making process, and the optimal decision (shown in Figure 3) as per the outcome generated by the tree here is Dapagliflozin due to minimized cost and reduced side effects.

\section{Optimal decision}

The optimal drug recommended by the Palisade software was Dapagliflozin due to its reduced cost and side effects.

\section{Sensitivity analysis}

We conducted a one-way deterministic sensitivity analysis in Palisade software to assess the robustness of the model around the key parameters. We focused more on the cost and $\mathrm{HbA1c}$ in our sensitivity analysis as it can affect the ICER values the most. The results of the sensitivity analysis were generated as spider and tornado graphs (shown in Figure 4).

Table 3. Cost-effectiveness plane of Dapagliflozin and Canagliflozin.

\begin{tabular}{|c|c|c|c|c|c|c|c|c|c|}
\hline 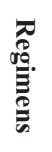 & Options/drugs & 丞 & $\begin{array}{c}\text { (vs. Control) } \\
\text { Incremental cost }\end{array}$ & $\begin{array}{c}\text { (vs. Control) } \\
\text { Incremental } \\
\text { effect }\end{array}$ & $\overline{\widehat{\widehat{T}}}$ & Q1 & Q2 & 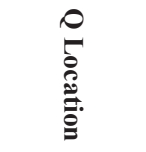 & $\begin{array}{c}\text { Label for } \\
\text { bar graph } \\
\text { (name }+ \\
\text { note 2) }\end{array}$ \\
\hline A & Dapagliflozin (Control) & 219 & 0 & 0.00 & 0.0 & Control & Control & Intersection & A-Control \\
\hline B & Canagliflozin (Comparison) & 401 & 1,264 & -0.19 & $-6,686$ & Dominated & Reject & North West & B-Reject \\
\hline
\end{tabular}




\section{Tornado Graph of ICER}

Impact by Input

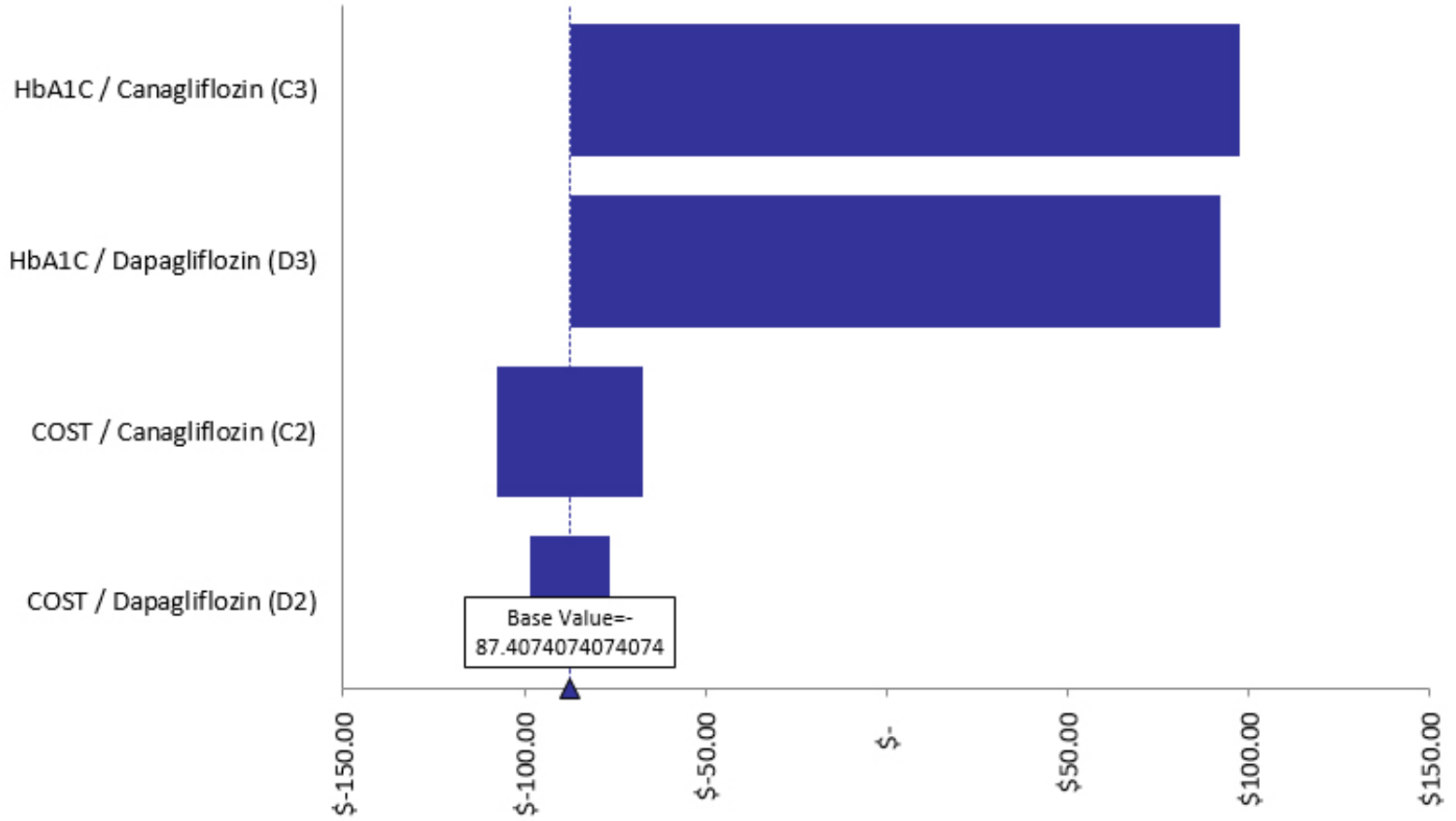

Value of ICER

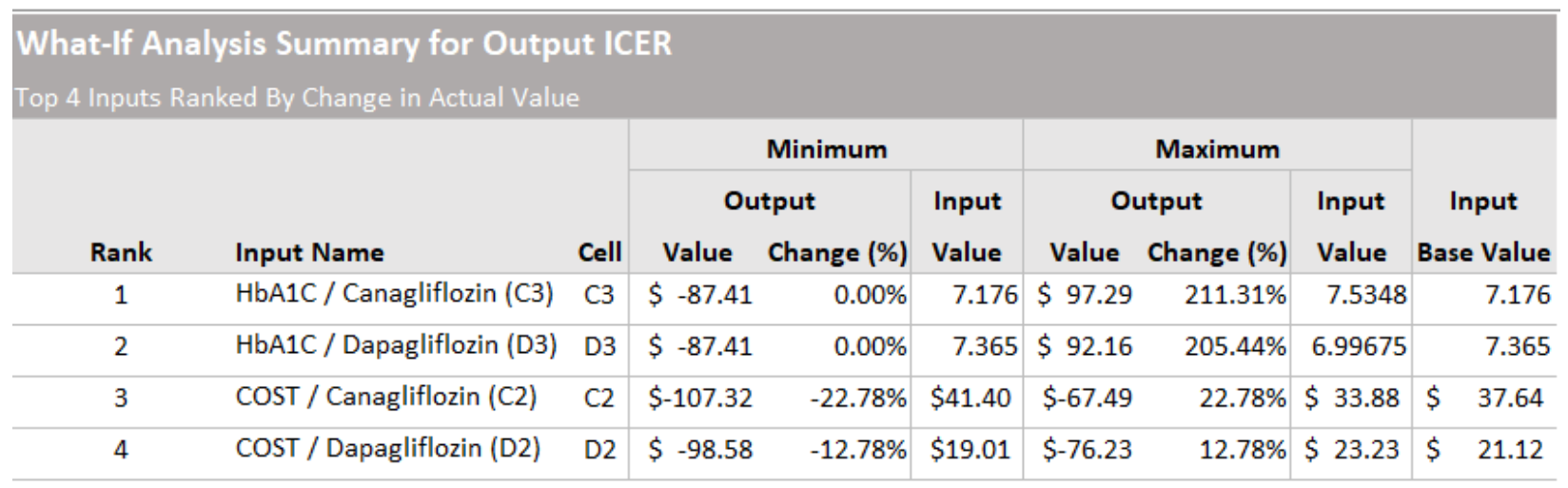

Figure 4. Tornado sensitivity graph.

It is ranked by change in actual value which means HbAlc of Canagliflozin can influence the output which is the ICER the most and the cost of Dapagliflozin can influence the ICER the least.

The spider graph shows the variation of each input. The value is set over a variation range of $-10 \%$ and $+10 \%$. The table above represents the variations shown in the spider sensitivity graph (shown in Figure 5). The result of the sensitivity analysis illustrated that $\mathrm{HbA} 1 \mathrm{c}$ of Canagliflozin can have the maximum impact on the ICER.

\section{DISCUSSION}

The present study was conducted to compare and analyze the cost and effectiveness of drugs Dapagliflozin and Canagliflozin for the treatment of type 2 diabetes mellitus by using the ECHO models.

The prevalence of diabetes appears to be higher in the 27-55 year age which indicates the use of certain anti-diabetic regimens higher in this population similar to THE DECODA STUDY GROUP (DECODA Study Group, 2003). Our study also found that type 2 diabetes is more prevalent in men than in 


\begin{tabular}{|c|c|c|c|c|c|c|c|c|c|}
\hline \multicolumn{10}{|c|}{$\begin{array}{l}\text { What-If Analysis Summary for Output ICER } \\
\text { Top } 4 \text { Inputs Percent Change vs Output Percent Change }\end{array}$} \\
\hline \multirow[b]{2}{*}{ Input Name } & \multirow[b]{2}{*}{ Cell } & \multirow[b]{2}{*}{ Step } & \multicolumn{3}{|c|}{ Input Variation } & \multicolumn{4}{|c|}{ Output Variation } \\
\hline & & & Value & Change & Change $(\%)$ & & Value & Change & Change (\%) \\
\hline \multirow[t]{5}{*}{ COST / Canagliflozin (C2) } & \multirow[t]{5}{*}{$\mathrm{c} 2$} & 1 & 33.876 & -3.764 & $-10.00 \%$ & $\$$ & -67.49 & $\$ 19.92$ & $22.78 \%$ \\
\hline & & 2 & 35.758 & -1.882 & $-5.00 \%$ & $\$$ & -77.45 & $\$ \quad 9.96$ & $11.39 \%$ \\
\hline & & 3 & 37.64 & 0 & $0.00 \%$ & $\$$ & -87.41 & $\$$ & $0.00 \%$ \\
\hline & & 4 & 39.522 & 1.882 & $5.00 \%$ & $\$$ & -97.37 & $\$ \quad-9.96$ & $-11.39 \%$ \\
\hline & & 5 & 41.404 & 3.764 & $10.00 \%$ & & -107.32 & $\$-19.92$ & $-22.78 \%$ \\
\hline \multirow[t]{5}{*}{ COST / Dapagliflozin (D2) } & \multirow[t]{5}{*}{$\mathrm{D} 2$} & 1 & 19.008 & -2.112 & $-10.00 \%$ & $\$$ & -98.58 & $\$-11.17$ & $-12.78 \%$ \\
\hline & & 2 & 20.064 & -1.056 & $-5.00 \%$ & $\$$ & -92.99 & $\$ \quad-5.59$ & $-6.39 \%$ \\
\hline & & 3 & 21.12 & 0 & $0.00 \%$ & $\$$ & -87.41 & $\$$ & $0.00 \%$ \\
\hline & & 4 & 22.176 & 1.056 & $5.00 \%$ & $\$$ & -81.82 & 5.59 & $6.39 \%$ \\
\hline & & 5 & 23.232 & 2.112 & $10.00 \%$ & $\$$ & -76.23 & $\$ 11.17$ & $12.78 \%$ \\
\hline \multirow[t]{5}{*}{ HbA1C / Canagliflozin (C3) } & \multirow[t]{5}{*}{$\mathrm{c3}$} & 1 & 6.4584 & -0.7176 & $-10.00 \%$ & $\$$ & -18.22 & $\$ 69.19$ & $79.15 \%$ \\
\hline & & 2 & 6.8172 & -0.3588 & $-5.00 \%$ & $\$$ & -30.16 & $\$ 57.25$ & $65.50 \%$ \\
\hline & & 3 & 7.176 & 0 & $0.00 \%$ & $\$$ & -87.41 & $\$ \quad-$ & $0.00 \%$ \\
\hline & & 4 & 7.5348 & 0.3588 & $5.00 \%$ & $\$$ & 97.29 & $\$ 184.70$ & $211.31 \%$ \\
\hline & & 5 & 7.8936 & 0.7176 & $10.00 \%$ & $\$$ & 31.25 & $\$ 118.66$ & $135.75 \%$ \\
\hline \multirow[t]{5}{*}{ HbA1C / Dapagliflozin (D3) } & \multirow[t]{5}{*}{ D3 } & 1 & 6.6285 & -0.7365 & $-10.00 \%$ & $\$$ & 30.17 & $\$ 117.58$ & $134.52 \%$ \\
\hline & & 2 & 6.99675 & -0.36825 & $-5.00 \%$ & $\$$ & 92.16 & $\$ 179.57$ & $205.44 \%$ \\
\hline & & 3 & 7.365 & o & $0.00 \%$ & $\$$ & -87.41 & $\$ \quad-$ & $0.00 \%$ \\
\hline & & 4 & 7.73325 & 0.36825 & $5.00 \%$ & $\$$ & -29.65 & $\$ 57.76$ & $66.08 \%$ \\
\hline & & 5 & 8.1015 & 0.7365 & $10.00 \%$ & $\$$ & -17.85 & $\$ 69.56$ & $79.58 \%$ \\
\hline
\end{tabular}

\section{Spider Graph of ICER}

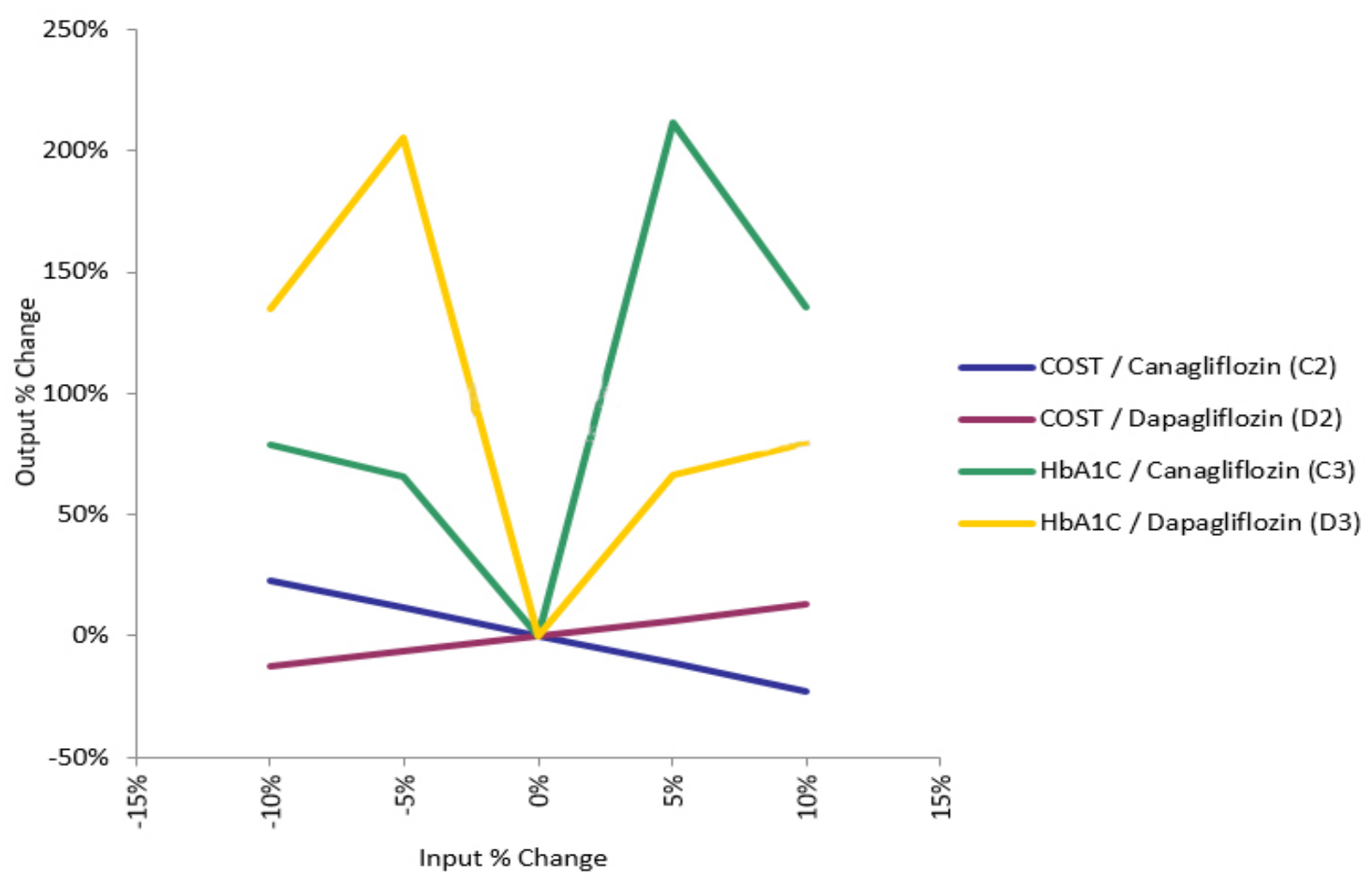

Figure 5. Spider sensitivity graph. 
women according to the gender classification, which is similar to Alexandra's study (Kautzky-Willer et al., 2016).

For measuring the effectiveness of drugs in terms of clinical outcomes HbAlc was considered. Both the groups achieved the expected target HbA1c (A1C targets of $<7 \%$ or $53 \mathrm{mmol} / \mathrm{mol}$ ). However, the drug Canagliflozin shows slight improvement that is 0.189 in the effectiveness arm compared to Dapagliflozin similar to studies by Hou et al. (2019) and Neslusan et al. (2018).

Global surveillance shows Dollar (\$) is the standard world's principle currency for supporting economic analysis. As per WHO CHOICE recommendations, the direct costs of the medications are measured by using MPV for the selected treatment regimens to conduct the pharmacoeconomic analysis.

To measure the economical outcome, the MPV of Canagliflozin (1 brand) and Dapagliflozin (three brands) was calculated for India. The direct and indirect cost associated with both the treatment groups was also calculated. The total cost was found to be more with Canagliflozin treated patients due to the increased cost of treatment of side effects and associated hospitalizations than patients who took Dapagliflozin, and Dapagliflozin showed the least cost spent by the patients than Canagliflozin treated patients which was contradictory with the study conducted by Hou et al. (2019).

The DSC-R Scale was used to measure the level of disease-related complications and comorbid conditions in the study population and Humanistic outcomes were measured as QALYs. The measure of health outcomes gained by the patient for a particular treatment regimen was determined by using the MDS of the DSC-R. None of the selected treatment regimens reached the expected outcomes as full health, to the rating scale of $0-1$. This further demonstrates that both the drugs produce only partial health outcomes. But Better QALY results were shown by Dapagliflozin and which was contradictory to the study by Hou et al. (2019).

These results also signify that in patients with type $2 \mathrm{DM}$ treatments the clinical outcomes are affected by multiple factors like medication adherence, improper diet plan, and selection of regimens. These issues need to be considered and analyzed in future to understand the correlation between humanistic and other economical outcomes.

In our study, CEA was done with the selected antidiabetic treatment regimens, Dapagliflozin was taken as the control group (A) and Canagliflozin was taken as the comparison group (B), and finally the comparison group (B) was rejected and the control group (A) was accepted.

A decision tree model was generated with the Palisade software using the data collected for the study purpose from various community pharmacies across Kerala. Considering all these parameters the direct cost, the indirect cost, and the probability, the software generates an optimal decision for Dapagliflozin which aids the decision-making process.

Sensitivity analysis also supported the robustness of estimated results, showing that the effectiveness of the drug is an important parameter when determining the CEA. The result of the sensitivity analysis illustrated that HbA1c of Canagliflozin can have the maximum impact on the ICER. The limitation of the analysis is the inadequate head-to-head clinical evidence for Dapagliflozin and Canagliflozin in patients with type 2 diabetes.

Similar studies on CEA of Canagliflozin and Dapagliflozin added to metformin were conducted abroad showed that Canagliflozin added with metformin was more cost effective than Dapagliflozin added to metformin because the price of Canagliflozin is lesser in these countries (Hou et al., 2019; Neslusan et al., 2018).

\section{LIMITATIONS}

The sample size was found to be less in our pharmacoeconomic analysis and an equal number of patients was not added for the comparison of regimens because Patients taking these drugs were very few when compared to other anti-diabetic drugs. ECH-Outcomes can be affected by various factors and that was accounted for in the analysis, and the humanistic outcomes are often influenced by the patient perceptions about their health status and overall wellbeing.

\section{CONCLUSION}

In this study, Dapagliflozin and Canagliflozin, type 2 anti-diabetic treatments were compared for clinical, economical, and humanistic outcome assessments. Target HbA1c (A1C targets of was achieved by both the antidiabetic treatment regimens but better results were shown in Canagliflozin treated patients. Average treatment cost per year was found to be higher with Canagliflozin than Dapagliflozin. Humanistic outcome analysis results showed both the antidiabetic regimens produced only partial health status QALY but better results were shown with Dapagliflozin. Additionally, CEA analysis shows Dapagliflozin as more effective and less expensive than comparison with Canagliflozin which falls on Quadrant four North West Position.

According to the results of this study, Dapagliflozin could be considered as a cost-effective regimen. We assume that the economical, and clinical outcomes of Pharmacoeconomics were affected by various other factors like medication adherence, affordability, appropriateness of regimens, and other social and cultural factors. Further studies should be conducted on these matters to understand and improve the QALY and also to reduce the burden of disease of type 2 diabetic patients.

\section{ACKNOWLEDGMENT}

The authors would like to express our profound gratitude to Dr. Santosh Kumar, for his valuable guidance and continuous encouragement.

\section{AUTHOR CONTRIBUTIONS}

All authors made substantial contributions to conception and design, acquisition of data, or analysis and interpretation of data; took part in drafting the article or revising it critically for important intellectual content; agreed to submit to the current journal; gave final approval of the version to be published; and agree to be accountable for all aspects of the work. All the authors are eligible to be an author as per the international committee of medical journal editors (ICMJE) requirements/guidelines. 


\section{FUNDING}

There is no funding to report.

\section{CONFLICTS OF INTEREST}

The authors report no financial or any other conflicts of interest in this work.

\section{DATA AVAILABILITY}

research article.

All data generated and analyzed are included within this

\section{PUBLISHER'S NOTE}

This journal remains neutral with regard to jurisdictional claims in published institutional affiliation.

\section{ABBREVIATIONS}

DM Diabetes Mellitus

SGLT2 Sodium-glucose co-transporter-2

DSC-R Diabetes Symptom Checklist-Revised

HbA1c Glycated hemoglobin

AE Adverse Event

\section{REFERENCES}

Akari S, Mateti UV, Kunduru BR. Health-care cost of diabetes in South India: a cost of illness study. J Res Pharm Pract, 2013; 2:114-7.

Anjana RM, Pradeepa R, Deepa M, Datta M, Sudha V, Unnikrishnan R, Bhansali A, Joshi PP, Yajnik CS, Dhandhania VK. Prevalence of diabetes and prediabetes (impaired fasting glucose and/or impaired glucose tolerance) in urban and rural India: phase I results of the Indian Council of Medical Research-INdia DIABetes (ICMR-INDIAB) study. Diabetologia, 2011; 54:3022-7.

DECODA Study Group. Age-and sex-specific prevalence of diabetes and impaired glucose regulation in 11 Asian cohorts. Diabetes Care, 2003; 26(6):1770-80.
Hou X, Wan X, Wu B. Cost-effectiveness of Canagliflozin versus Dapagliflozin added to metformin in patients with type 2 diabetes in China. Front Pharmacol, 2019; 10:480.

Kautzky-Willer A, Harreiter J, Pacini G. Sex and gender differences in risk, pathophysiology and complications of type 2 diabetes mellitus. Endocrine Rev, 2016; 37:278-316.

Li J, Gong Y, Li C, Lu Y, Liu Y, Shao Y. Long-term efficacy and safety of sodium-glucose cotransporter-2 inhibitors as add-on to metformin treatment in the management of type 2 diabetes mellitus: a meta-analysis. Medicine, 2017; 96(27):e7201.

Neslusan C, Teschemaker A, Willis M, Johansen P, Vo L. Costeffectiveness analysis of canagliflozin $300 \mathrm{mg}$ versus dapagliflozin $10 \mathrm{mg}$ added to metformin in patients with type 2 diabetes in the United States. Diabetes Ther, 2018; 9(2):565-81.

Pereira MJ, Eriksson JW. Emerging role of SGLT-2 inhibitors for the treatment of obesity. Drugs, 2019; 79(3):219-30.

Rabizadeh S, Nakhjavani M, Esteghamati A. Cardiovascular and renal benefits of SGLT2 inhibitors: a narrative review. Int J Endocrinol Metab, 2019; 17(2):e84353.

The Blonde Pharmacist. BCPS 2013: some pharmacoeconomics [Online]. 2019. Available via http://www.theblondepharmacist.com/ blog/2013/04/10/ pharmacoeconomics (Accessed 26 April 2019).

\section{How to cite this article:}

Sadagoban GK, Shaji JR, Premnath B, Siby AR, Borra SS, Ayilya M, Parthasarathy AK. Cost-effectiveness analysis of Dapagliflozin versus Canagliflozin in treatment of type 2 diabetes mellitus. J Appl Pharm Sci, 2022; 12(03): 171-178. 